\title{
Performance Analysis and Resource Allocation in MIMO-OFDM Systems
}

\author{
Prof. Muhamad Angriawan \\ Department of Computer Engineering, IRC Russia \\ muhamadaggriawan@mail.ru
}

\begin{tabular}{|l|l|}
\hline Article History & \multicolumn{1}{c|}{ Abstract } \\
$\begin{array}{l}\text { Article Submission } \\
10 \text { February } 2016 \\
\text { Revised Submission } \\
27 \text { April } 2016 \\
\text { Article Accepted }\end{array}$ & $\begin{array}{l}\text { The paper deals with the analysis of the vital performance plot of SNR and BER in } \\
\text { BER plot with respect to it is also studied using the simulation outputs. The three } \\
\text { types of fading channels are also analysed. It is also seen that the presence of } \\
\text { Article Published } \\
\text { 30 June 2016 }\end{array}$ \\
$\begin{array}{l}\text { diversity and other schemes like Maximal Ratio Combining, selection combining, } \\
\text { techniques and the superiority of the Monte Carlo optimization to the theoretical } \\
\text { system without optimisation is also clearly visualised. The paper also deals with } \\
\text { resource allocation in MIMO-OFDM systems. The advantage of the constant } \\
\text { envelope OFDM over OFDM is achieved. Also the power allocation using water- } \\
\text { filling algorithm and bandwidth-power product minimisation is compared using the } \\
\text { obtained results. } \\
\text { Keywords: MIMO, OFDM, LTE, Bandwidth-Power Product, SNR, power allocation. }\end{array}$ \\
\hline
\end{tabular}

\section{Introduction}

The reliability of any communication system is significantly dependent on the channel fading models. However, the significance of it is higher in the case of wireless communication systems than in the case of wired systems. These channels can be modeled based on their characteristics as a probabilistic distribution function [1][2]. An Additive White Gaussian (AWGN) channel is generally used to model the channel response of a wired system. Rayleigh and Rician fading channels used to model the channel response of wireless communication systems. Rayleigh fading channel model is used in cases involving the lack of a LoS component whereas the Rician fading model is generally used when a LoS component is available between the transmitter and receiver. The general expression for a MIMO system model is,

$$
\mathrm{Y}=\mathrm{Hx}+\mathrm{N}
$$

where $\mathrm{Y}$ is the matrix of the received signal at the receiver, $\mathrm{H}$ is the channel matrix of dimension $\mathrm{M}_{\mathrm{R}}$ receive antennas and $\mathrm{M}_{\mathrm{T}}$ transmit antennas., $\mathrm{x}$ is the signal matrix and $\mathrm{N}$ denotes the Noise Power. Some boundaries are physical antenna spacing, multiple wavelengths, antenna separation at the receiver, antenna design and algorithm techniques. In order to maximize the performance of the wireless systems, several combining techniques are used at the receiver. Some of the techniques are Maximal Ratio Combining (MRC), Equal Gain Combining (EGC), etc.

The wireless channel may also be frequency flat or frequency selective fading channel. But the usage of modern schemes like space time coding, OFDM divide the frequency selective channel into a number of frequency flat sub-channels. The relay networks present in between the transmitter and receiver provide the communication between them. Various theoretical and optimization schemes are used in the relay networks to increase the overall efficiency of the system [3][4]. The performance metrics of the system include the capacity, outage probability, Signal to Noise Ratio (SNR), symbol or Bit Error Rate (BER).

The cyclic prefix added might be either a part of the data or 0s. The addition of cyclic prefix greatly decreases the ISI. Hence OFDM decomposes $\mathrm{n}$ independent frequency selective fading channels into $\mathrm{n}$ independent frequency flat channels. The combination of the MIMO concept with the OFDM scheme is the key technology of $4 \mathrm{G}$ wireless standard for mobile communication and provides efficient multimedia support [5]. 
The remainder of the paper is organized as follows. Section II deals with the description of the system model. Section III deals with problem definition of the MIMO-OFDM system, Section IV deals with the adaptive resource allocation. Section V shows the simulation results and corresponding inferences. Section VI concludes the paper.

\section{System Model}

The initial part of this section deals with the system model of a single user multi relay system. The system model has three main elements namely: Base station, User equipment and Relay networks. Base Station, in mobile communication is generally the stationary component that provides service to the mobile devices or users. It is monitored by the service provider and installed to operate in a particular bandwidth and support a number of users [6][7]. The signal power is determined by the base station. The area covered by a base station is called a cell. A cell may have more than one sector antenna. A group of cells combine to form a cluster.

User Equipment is the mobile device used by the user to acquire the services of the base station or service provider [8][9]. It is transceiver that can both transmit and receive. It can communicate with other mobile devices. The received signal strength determined by the Signal to Noise ratio plays a vital role here. Relay networks are one or more elements present in between the base station and user equipment (in mobile communication). They lie in between two communicating devices and help in establishing and maintaining the connection [10]. A communication system may have one or more relays. Systems without relays are possible but are comparatively inefficient.

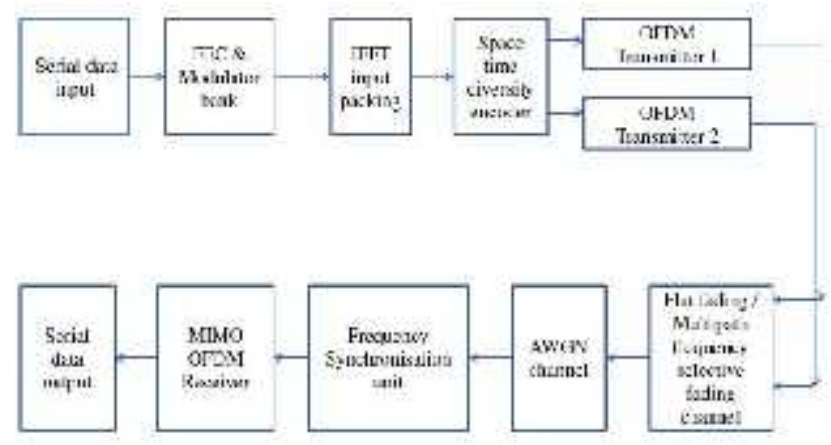

Fig 1: Scenario diagram of an OFDM system

A system may support single user or multiuser communication. A single user system is one in which the entire features of the communication system is utilized by a single user at a point of time. A multiuser system is one in which the features of a system can be simultaneously utilized or shared by more than one user. It resembles a single user MIMO system. The performance analysis (i.e.) Signal to Noise Ratio (SNR) and Bit Error Rate (BER) plots will be done later in Section V. The second part of this section combines the system model of MIMO and OFDM setup. The problems associated with the OFDM model and the Constant Envelope OFDM also called as CE-OFDM is also described in the following sections.

\section{Proposed Adaptive Resource Allocation Scheme}

OFDM is highly sensitive to synchronisation errors since it has overlapping spectra of subcarriers. This can be eliminated by using proper synchronising pulses at the transmitter and receiver. However, the large fading channels can prevent an optimum solution for this problem. An optimum power is essential to maintain the QoS in terms of the channel data rate and the error free rate or BER (Bit Error Rate). But a high Peak to Average Power Ratio can cause the inefficient performance of the components associated with the communication system. Any system must provide good throughput in order to minimise the loss of data and hence prevent retransmission and also not often test the error correcting capability of the block at the receiver. 
Even some of the adaptive algorithms function at optimal allocation of one resource at the expense of the other. The water-filling solution attempts to use as much bandwidth as possible to minimize the power thus resulting in a waste of spectral footprint. Resource Allocation is one of the important aspects of any communication system. Some of the resources to be allocated are:- Subcarriers, bits, Power and Bandwidth. The resource allocation is divided into two categories namely: Fixed and Dynamic Resource allocation.

In Fixed Resource Allocation, the resources are pre-allocated to the user and can be made available to the corresponding user during the system's runtime until it is re-allocated. The users have resources dedicated to them. But the usage by the users is not the same and maximum all the time. The usage may increase during peak hours or in a particular hotspot region. Some users may remain idle most of the time, whereas some users may not find their channel sufficient for their use. In all these cases, fixed resource assignment strategy does not provide an efficient and optimal solution.

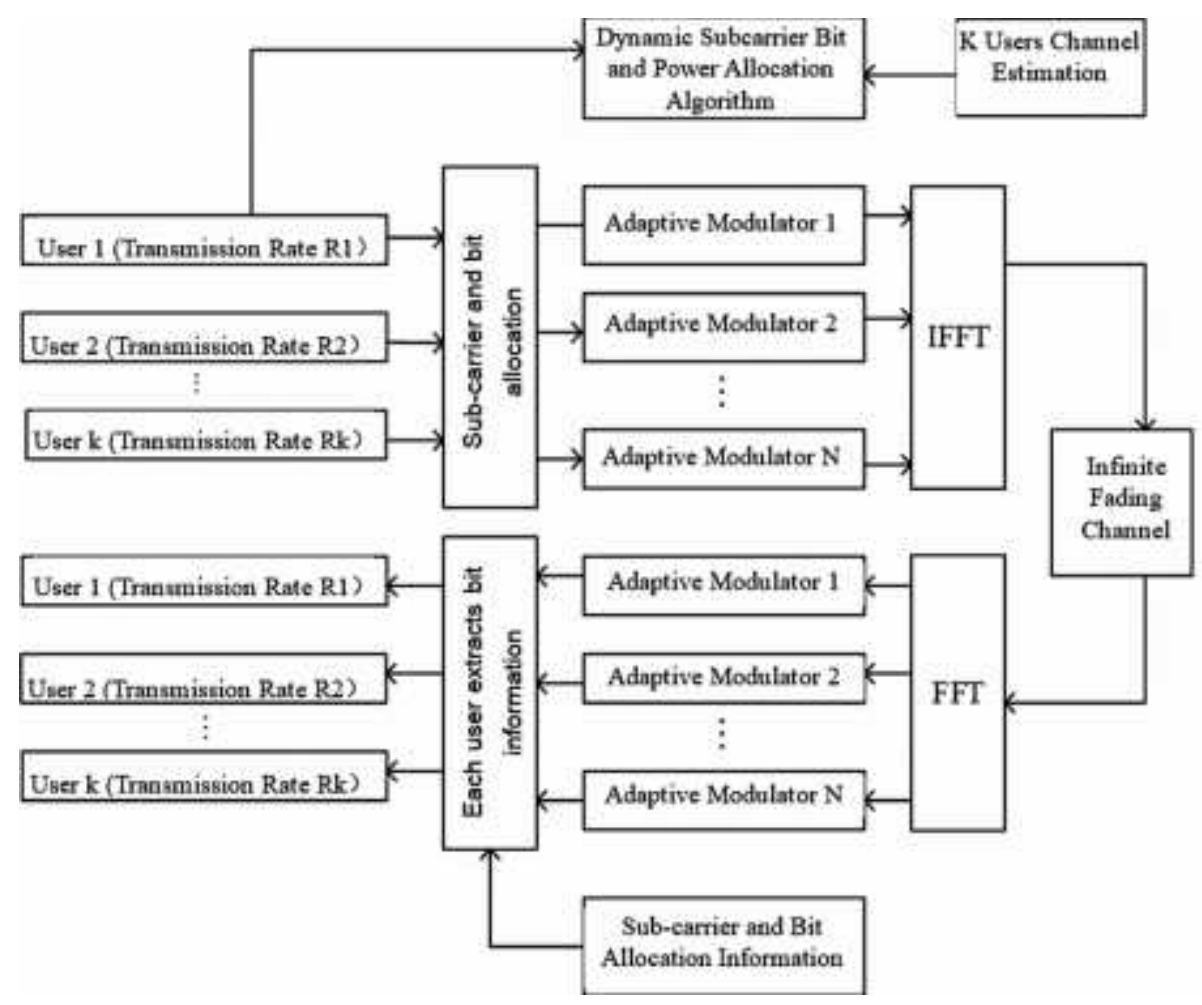

Fig 3: Proposed adaptive resource allocation scheme for OFDM system

In Dynamic Resource Allocation, the resources are allocated based on the need at the time of allocation. It is dynamic and varying (i.e.) the same resource may not be allocated to the same user at all times. Based on the statistics and availability and usage of the system by a user, the resources are allocated. There are generally two types of dynamic resource allocation techniques namely: Margin and Rate Adaptive. The MA technique achieves minimum overall transmit power with the constraint of user's data rate or BER. The RA technique maximizes user's error free capacity with the constraint of the total transmits power.

\section{Simulation Results}

The former part of this section deals with the performance analysis of MIMO system. The SNR vs BER plots for the various channel models, significance of diversity, Monte-carlo optimization and its comparison with theoretical plots, throughput of LTE system are analysed based on their plots. The latter part of this section deals with the comparison of OFDM with CE-OFDM. The comparison of water-filling algorithm for power allocation and bandwidth-power product minimization is also done in this part. The conventional fading channels are shown in figure 4. 


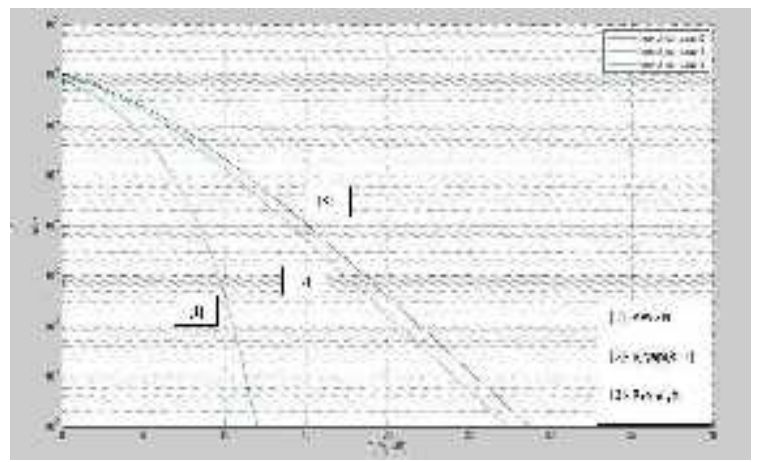

Fig 4: Comparison of fading channels

Figure 4 depicts the theoretical output of the variation of BER with SNR for the three fading models. It is seen that, for the AWGN channel, a good BER of 10-8 is obtained for an SNR of about $12 \mathrm{~dB}$. In case of Rician fading (2), a higher SNR of more than $27 \mathrm{~dB}$ is required to obtain the same BER. The $\mathrm{k}$ factor of Rician was considered to be 1 . In case of Rayleigh fading (3), an SNR of about $29 \mathrm{~dB}$ is required to obtain the same BER of 10-8. As the $\mathrm{k}$ factor is increased, the Rician fading approaches the AWGN fading. All these plots are obtained by giving the diversity order of 3 .

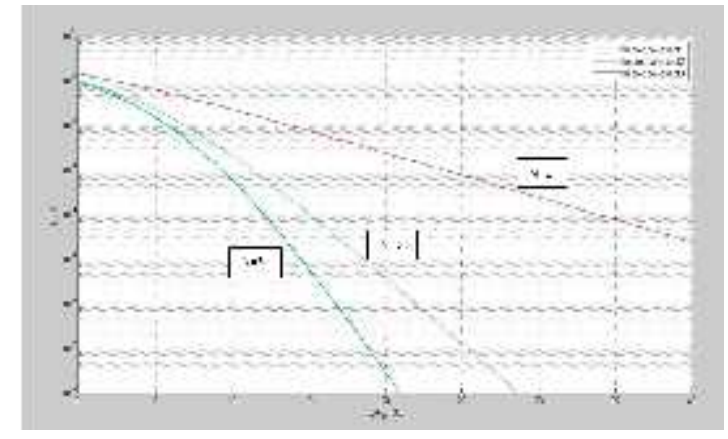

Fig 5: SNR vs BER for different diversity orders

Figure 5 shows the plot of SNR versus BER for three different values of diversity order, namely: $N=5,3$ and 1 . It is evident from the plot that a better BER is obtained for the same SNR as the diversity order is increased. N=1 is a SISO system with no diversity technique used. the other two are MIMO systems. For an SNR of $20 \mathrm{~dB}, \mathrm{~N}=1$ gives a BER greater than 10-3. For the same SNR, $\mathrm{N}=3$ gives a BER between 10-5 and 10-6. But in the case of $\mathrm{N}=5$, a very good BER of nearly $10-8$ is obtained for $20 \mathrm{~dB}$. This proves that a better BER is obtained even for low SNRs when higher diversity orders are used. Note that this plot and all the plots henceforth are for Rayleigh fading channels, since the design for MIMO system is to be done for the worst case.

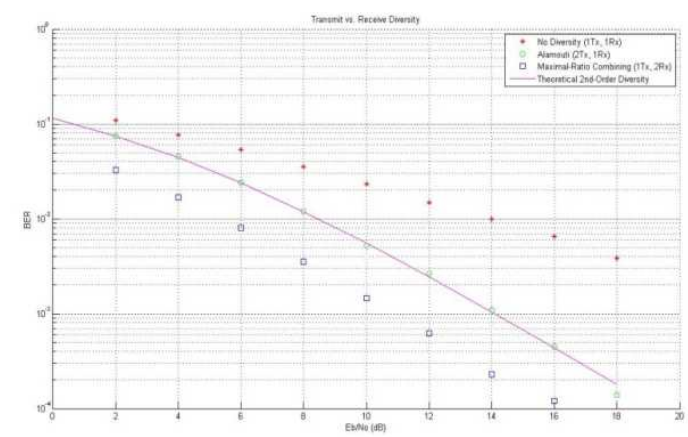

Fig 6: Demonstration of diversity significance 
Figure 6 shows the clear demonstration of the significance of the diversity scheme. It includes the plot of SNR and BER for:- No diversity(1 Tx, $1 \mathrm{Rx})$, Alamouti(2 Tx, $1 \mathrm{Rx})$, Maximal Ratio Combining(1 Tx, $2 \mathrm{Rx})$ and theoretical second order diversity. It is seen that, as discussed above, the plot with no diversity doesn't have an appreciable performance compared to the others. The alamouti scheme and the theoretical second order diversity almost overlie each other. The maximal ratio combining is used in cases where the signal is received by multiple antennas and selection is done based on the signal received with the maximum SNR. The usage of this technique further improves the performance of the system and hence a better BER is obtained.

In addition to the maximal ratio combining technique, various other techniques like Equal Gain combining, selection combining, etc can also be used. These techniques show better performance according to the environment of their installation and some other factors. Fig 7 shows the comparison between the theoretical performance curve and that obtained through Monte Carlo optimisation. Initially for lower values of SNR, the difference between the plots is not significant. But as the SNR values increase beyond $10 \mathrm{~dB}$, the difference becomes significant. A very high comparative performance is seen with Monte carlo optimization than the theoretical case without optimization.

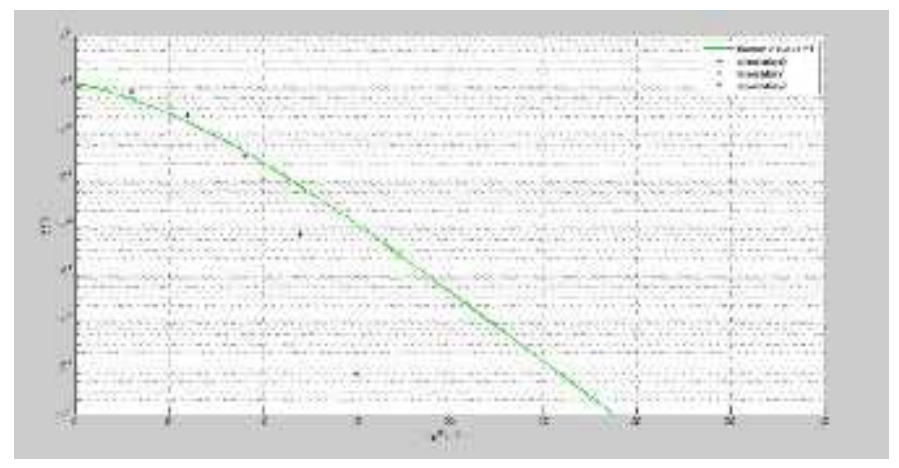

Fig 7: Monte carlo and theoretical Rayleigh plot

The power spectrum in $\mathrm{dBm}$ and the real part of the OFDM signal are calculated and analysed for the general OFDM system. In order to achieve a better reduction in PAPR, the Constant Envelope OFDM (CE-OFDM) system is implemented and the corresponding outputs are analysed in figure 8 .

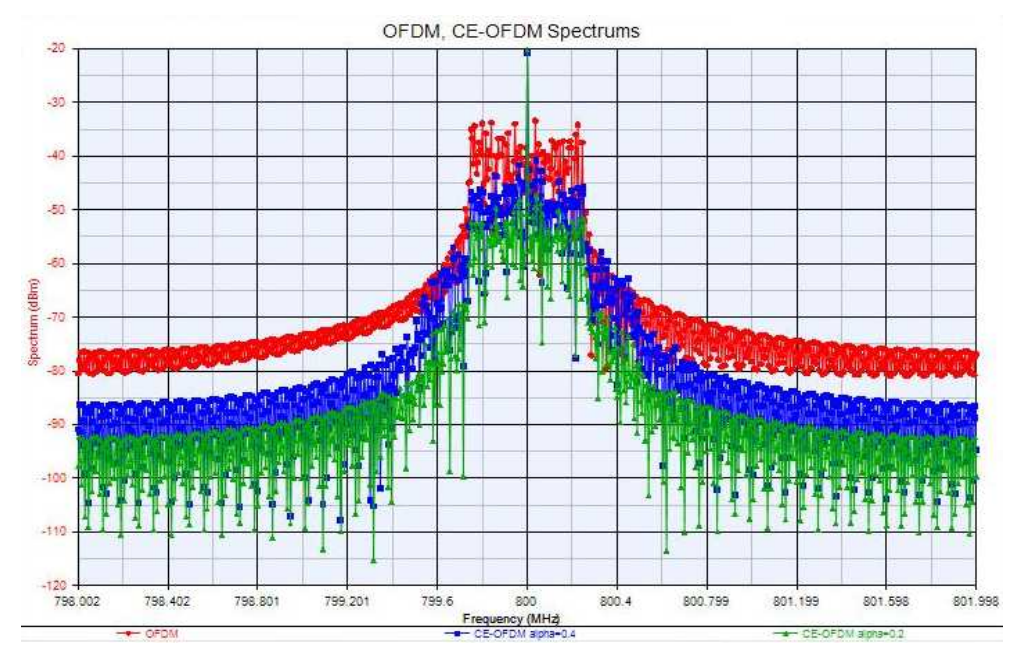

Fig 8: Frequency Vs Power spectrum 
As shown in Fig. 8, the red colored graph depicts the plot of the real part of the OFDM signal. The blue colored graph depicts the plot of the real part of the CE-OFDM signal. It is inferred from the graph that the variation of the OFDM (red) signal is more than that of the blue signal. This results in a higher PAPR ratio of the OFDM signal. However, the blue signal has fewer fluctuations and hence results in lower PAPR compared to the OFDM signal. Therefore, CE-OFDM system can be used instead of OFDM system in cases requiring minimum PAPR ratio.

In Fig.9, the red coloured graph depicts the Power spectrum of the OFDM signal with respect to the frequency. The other two coloured graphs depict the Power spectrum of the CE-OFDM signal. The blue colored graph illustrates the case where the gain alpha is 0.4 and the green colored graph illustrates the case where the value of alpha is 0.2 . The frequency range is from $798 \mathrm{MHz}$ to $802 \mathrm{MHz}$. The y axis is the power spectrum in $\mathrm{dBm}$. It is evident that the red signal has the highest value of the spectrum at majority of the frequencies followed by the blue and green signals respectively. Hence CE-OFDM is superior to OFDM in terms of conserving the power spectrum.

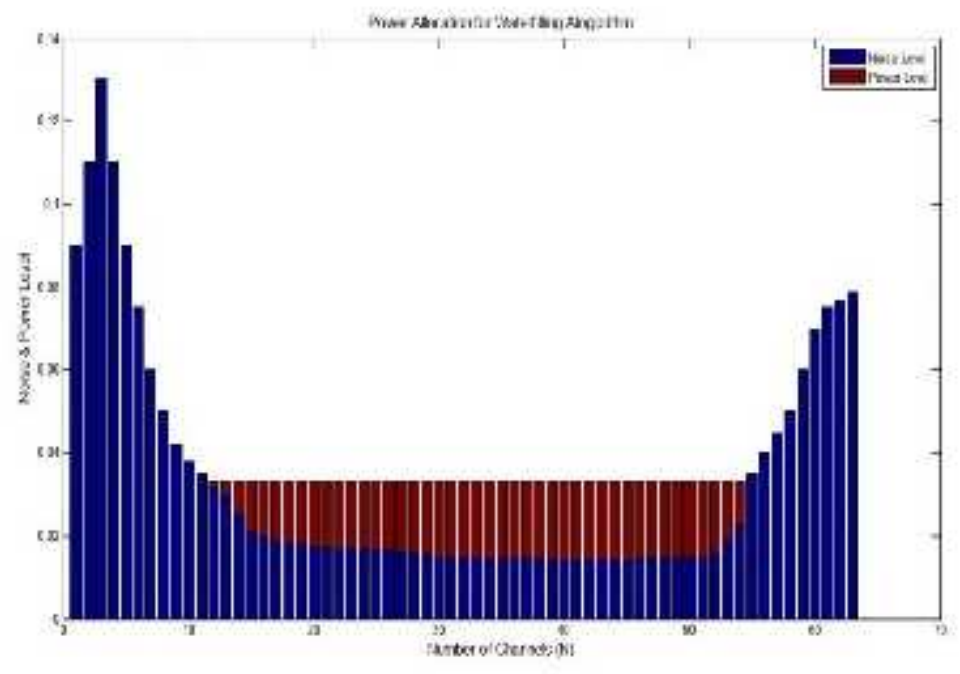

Fig 9: Power allocation using water-filling algorithm

The bandwidth-power product minimization method of power allocation. The power is allocated only for the channels whose bandwidth-power product is minimum. Hence it occupies fewer channels and thereby lesser bandwidth. A comparatively higher power is also allocated to these channels.

\section{Conclusion}

The performance of multiuser multiple antenna amplify and forward networks over Rayleigh fading channel was analysed. Moreover, the performance variation in the three types of fading channels namely: AWGN, Rayleigh and Rician is studied. Based on the simulation results obtained, variance performance metrics and plots were obtained. The significance of the diversity order was also proved. The importance of the diversity schemes like Alamouti, maximal ratio combining also were viewed through the plot. It is also seen that a better performance is obtained for increase in the diversity order. The variation of BER with SNR is studied under various cases. The optimisation scheme of Monte Carlo method was used and the conclusions are made from the simulation results obtained. The efficiency of the Monte Carlo method is inferred. OFDM is a promising technology that is a key technology alongside MIMO in $4 \mathrm{G}$ communication. The difference between the performances of OFDM and CE-OFDM is studied based on the results obtained. The spectrum obtained justifies the inference. The efficiency of Bandwidth-Power minimization in terms of bandwidth efficiency over traditional water-filling algorithm is analysed. 


\section{References}

[1] Yuzhen Huang, Fawaz Al-Qahtani,Caijun Zhong, Qihui Wu, Jinlong Wang and Hussein Alnuweiri, "Performance Analysis of Multiuser Multiple Antenna Relaying Networks with Co-Channel Interference and Feedback Delay", IEEE Transactions on Communications, Vol.62, No.1, January 2014.

[2] Yahia Tachwali, Brandon F. Lo, Ian F. Akyildiz, and Ramon Agusti, "Multiuser Resource Allocation Optimization Using Bandwidth-Power Product in Cognitive Radio Networks", IEEE Journal on selected areas in Communications, vol. 31, No. 3, March 2013.

[3] Jian Xu, Sang-Jin Lee, Woo-Seok Kang and Jong-Soo Seo, "Resource Allocation for MIMO-OFDM Based Wireless Multicast Systems", IEEE Transactions on Broadcasting, vol. 56, No. 1, March 2010.

[4] Farshad Shams, Giacomo Bacci and Marco Luise, "A survey on resource allocation techniques in OFDM(A) networks", ELSEVIER, Computer Networks 65 (2014) 129-150.

[5] B. Hassibi, B. Hochwald, Linear dispersion codes, in: Intern. Sympo. on Information Theory (ISIT), Washington, DC, p. 325.

[6] S. Chouhan and A. Sharma, "Performance analysis of STBC coded MIMO-OFDM system for WIMAX (IEEE 802.16) systems," 2015 International Conference on Innovations in Information, Embedded and Communication Systems (ICIIECS), Coimbatore, 2015, pp. 1-6, doi: 10.1109/ICIIECS.2015.7193233.

[7] R. D. Cajote, W. Ruangsang, S. Aramvith, P. Prapinmongkolkarn, Y. Miyanaga and T. Onoye, "Framework of surveillance video analysis and transmission system using background modeling and MIMO-OFDM," 2015 IEEE International Conference on Digital Signal Processing (DSP), Singapore, 2015, pp. 1071-1075, doi: 10.1109/ICDSP.2015.7252043.

[8] S. Ü. Ercan and Ç. Kurnaz, "Investigation of blind and pilot based channel estimation performances in MIMO-OFDM system," 2015 23nd Signal Processing and Communications Applications Conference (SIU), Malatya, 2015, pp. 1869-1872, doi: 10.1109/SIU.2015.7130221.

[9] C. Bo, "A Novel Adaptive Bit and Power Allocation Algorithm with High Efficiency for MIMOOFDM System," 2015 Sixth International Conference on Intelligent Systems Design and Engineering Applications (ISDEA), Guiyang, 2015, pp. 44-47, doi: 10.1109/ISDEA.2015.21.

[10] H. Ryu and B. Kim, "Beam space MIMO-OFDM system based on ESPAR antenna," 2015 International Workshop on Antenna Technology (iWAT), Seoul, 2015, pp. 168-171, doi: 10.1109/IWAT.2015.7365364. 